\title{
Improving PSD of PPM-IR for UWB Signal Using Turbo Encoder
}

\section{Mohammed Jabbar*}

University of Babylon, Babylon, Iraq

\begin{abstract}
The repression of the spectral line is a matter of main attention in designing of Time Hopping Impulse Radio (TH-IR) Ultra-Wide Band (UWB) systems. This case has been usually processed by randomizing the locations of every pulse. This requires to employ of very long Pseudo Noise (PN) sequences to suppress the capabilities of spectral lines. This research aims to improve the Power Spectral Density (PSD) by constructing the proposed system using Turbo code technique inside MATLAB's Simulink. We found that our proposed system provides high performance than Conventional PPM-TH-IR in term of eliminating the spectral lines or make it smoother. We also found that the increasing in interleaver length and constraint length enhances PSD.
\end{abstract}

\section{Keywords: TC; MTC; PN generator; PPM; PSD; TH-IR; UWB}

\section{Introduction}

A wireless transmission system that employ a bandwidth more than twenty-five percent of a center frequency or greater than $1.5 \mathrm{GHz}$ called UWB technology [1]. Over the past few years, UWB acquired much attention as a possible candidate for wireless short-range communication. The Federal Communications Commission (FCC) allocated spectrum range (3.1-10.6) GHz for UWB implementations [2].

Signals belonging to this category in accordance with the FCC regulations must have a-10dB bandwidth which exceeds $0.5 \mathrm{GHz}$ of its partial bandwidth, where:

$$
\begin{gathered}
\eta=\frac{2(\mathcal{F H}-\mathcal{F} \ell)}{(\mathcal{F H}+\mathcal{F} \ell)} \\
\mathcal{B}_{-10}=\mathcal{F H}-\mathcal{F} \ell
\end{gathered}
$$

$F H$ and $F \ell$ are the high and low $-10 \mathrm{~dB}$ cutoff frequencies, respectively [3]. Impulse Radio (IR) is one of the most significant technique to UWB.IR is oftentimes utilized as an equivalent term of UWB and its have stream of pulses with low duty cycle $[3,4]$. The effectiveness of UWB systems appears mainly in their of work overlapping with existing systems. In other meaning, since UWB does not need a new spectrum, its investment can be parallel to existing systems, as shown in Figure 1. The transmitted power could be expressed by product of PSD and the bandwidth. Therefore, the system with acceptable transmitted power and large bandwidth allows to show very low PSD. The narrowband receiver could be observe the noise power of its own system bandwidth, which very small part of UWB bandwidth. Because of this small interference, frequency organizers around the world are permitting UWB radiations in the microwave system [5].

Some of current methods are used for UWB system. In this paper, TH-IR is used. To modulate the pulses with information, it can be accomplished using Pulse Position Modulation (PPM) [6,7].

\section{Conventional PPM TH-IR System}

The conventional PPM TH-IR System consists of five stages as shown in Figure 2.

The Pseudo Noise (PN) generator contains eighteen shift register which can generate a maximal length sequence $2^{18}-1$ bits. The output of the PN generator is fed to the bias circuit. The work of a bias circuit is to change the probability of one's and zero's and it act best with very long sequence. The convolutional encoder (CC) with rate $1 / 3$ used to control the code prior applying it to the PPM system in order to reduce the spectral lines [8]. Generally, CC map the input bit into $\mathrm{n}$ length output bits, these $\mathrm{n}$ bits are not only determined by the present bit input but also depend the previous bits, this increases the capability of the communication systems to be able to dominate a lossy transmission medium and enhance the randomness $[9,10]$. PPM system, a technique in which the timing of each pulse is changed to transmit information instead of changing amplitude. It is modulation section that uses the pulse position to point the bit that was sent instead of sending the whole bits. It also sometimes refers to the code transmitted without sending all the bits. So it is good in terms of power consumption [11]. The spectrum analyzer is used as the fifth stage to measure the magnitude of the input signal versus frequency.

\section{Parallel Concatenation of Convolutional Codes}

Turbo Code (TC) consist from just two CC at a rate $1 / 2$ and random interleaver (permutations) at the input of the second encoder. When using more than two parallel CC with random interleavers at the input of each encoder will improves the performance of TC, and called Multiple Turbo Code (MTC). The encoder used in this paper contains three recursive binary CC as shown in Figure 3 [12].

\section{Proposed PPM TH-IR System}

Figure 4 shows the proposed system. In order to improve system, TC and MTC are used with different interleaving length and constraint length. Also, the simulation results are compared with the conventional PPM TH-IR UWB.

The signal created by the system, shown in Figure 4 is depicted by the equation

$$
y(t)=\sum_{i} p\left(\mathrm{t}-i T_{\tilde{n}}-\gamma_{i} \mathrm{~T}_{\gamma}\right)
$$

*Corresponding author: Mohammed Jabbar, University of Babylon, Babylon, Iraq, Tel: 7801006256; E-mail: mohammedalsalihy@yahoo.com

Received November 23, 2017; Accepted January 08, 2017; Published January 15, 2018

Citation: Jabbar M (2018) Improving PSD of PPM-IR for UWB Signal Using Turbo Encoder. Ind Eng Manage S3: 004. doi: 10.4172/2169-0316.S3-004

Copyright: (๑) 2018 Jabbar M. This is an open-access article distributed under the terms of the Creative Commons Attribution License, which permits unrestricted use, distribution, and reproduction in any medium, provided the original author and source are credited. 


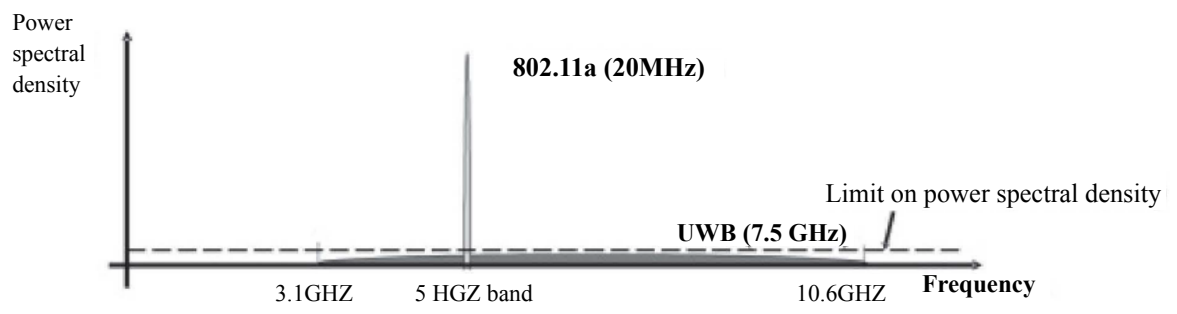

Figure 1: Interference between a UWB system and a narrowband (IEEE 802.11a) LAN.

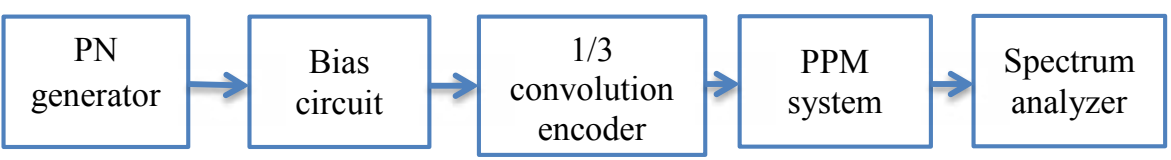

Figure 2: Conventional PPM TH-IR system block diagram.

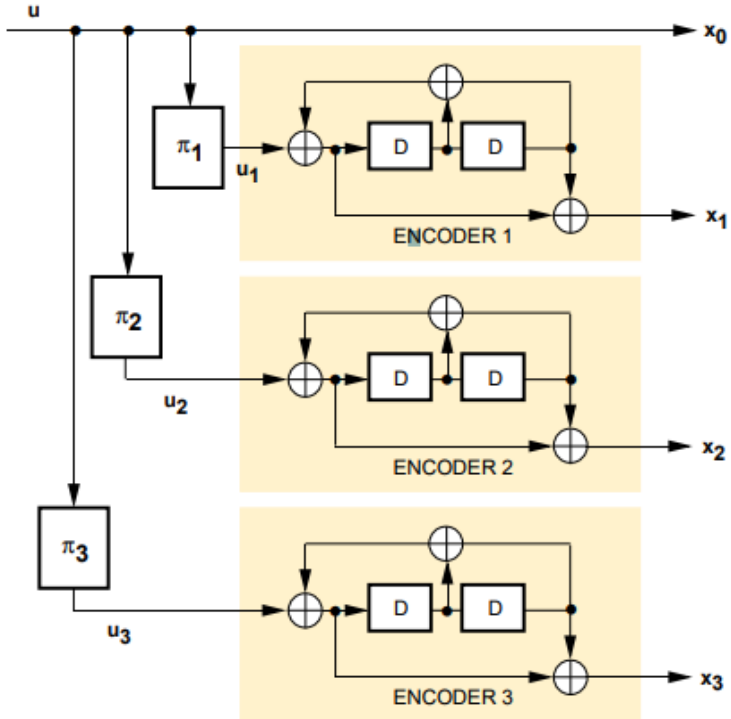

Figure 3: Encoder with three convolutional codes.

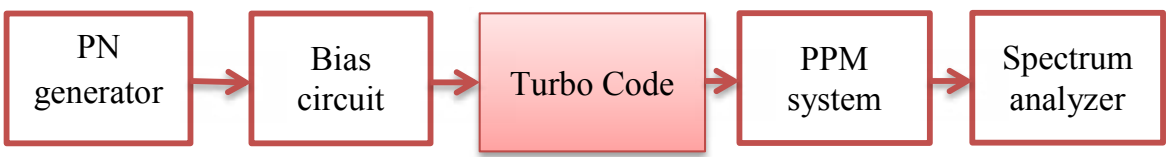

Figure 4: Proposed PPM TH-IR system block diagram.

Where $p(t)$ account for the pulse shape; $T$ is the mean pulse repetition rate; $\beta_{\mathrm{i}}$ is the $\mathrm{i}^{\text {th }}$ symbol from the encoder, and $\mathrm{T}_{\mathrm{\gamma}}$ is the PPM modulation index. Further, the PSD of $y(t)$ can be derived by using the results presented by the following formula:

$$
\begin{gathered}
\bar{H}(f)=\frac{1}{T_{\rho}}|P(f)|^{2}-\frac{1}{T_{r}}|P(f)|^{2}\left(\frac{1}{8}+\frac{2}{32} \sum_{j=1}^{8-1}\left(8-|j| \cos \left(2 \pi f j T_{\gamma}\right)\right)\right)+ \\
\frac{1}{T_{\rho}^{2}}\left(\frac{1}{8}+\frac{2}{32} \sum_{j=1}^{8-1}\left(8-|j| \cos \left(2 \pi f j T_{\gamma}\right)\right)\right) \sum_{-\infty}^{\infty} \delta\left(f-\frac{v}{T_{\rho}}\right)
\end{gathered}
$$

Where $\mathrm{P}(\mathrm{f})$ represents the Fourier transform of $\mathrm{P}(\mathrm{f})$. The value of $\mathrm{T}_{\gamma}$ is select to remove spectral lines as possible [13-15].

\section{Simulation Parameters}

The simulation parameters used for PPM TH-IR system are listed in Table 1.

\section{Simulation Results}

In this part, we will compare the conventional PPM TH-IR with 
proposed system in two scenarios. The same procedures have been repeated in an attempt to detect different codes that provide better PSD.

Figure 3 shows PSD obtained by using conventional PPM THIR system. It's clearly a continuous component of harmonics with a numerous spectral lines. This appearance may be explained by the scarcity of the randomness between consecutive pulses (Figure 5).

In the first scenario, $\mathrm{TC}$ is tested with different properties to achieve a smoother PSD as shown in Figures 6a-6d.

When Figure 5 is compared with Figures 6a-6d it can be observed that the spectral lines are better as well as some of them has been eliminated. In the second scenario, MTC is tested with different properties to improve PSD as shown in Figures $7 \mathrm{a}$ and $7 \mathrm{~b}$.

The results shown in Figures $7 \mathrm{a}$ and $7 \mathrm{~b}$ illustrate that MTC improve PSD more than the TC due to large randomness between consecutive pulses.

\section{Conclusion}

It is concluded that the proposed PPM-TH-IR system improved the PSD by eliminating the spectral lines that are achieved by increasing the randomness of the transmitted signal. As well as, when increasing the constraint length and/or interleaving length in TC, MTC, It's observed

\begin{tabular}{|l|r|}
\hline Parameters & Values \\
\hline Resolution bandwidth $(\mathrm{GHz})$ & 20 \\
\hline Source type & Pseudo Noise generator \\
\hline pulse repetition rate $(\mathrm{sec})$ & $5^{*} 10^{-10}$ \\
\hline CC & Rate $(\mathrm{R})=1 / 3$ \\
\hline TC and MTC & Constraint length $(\mathrm{K})=7$ \\
& Rate $(\mathrm{R}): 1 / 3$ for $\mathrm{TC} \& 1 / 4$ for $\mathrm{MTC}$ \\
\hline
\end{tabular}

Table 1: Simulation Parameters

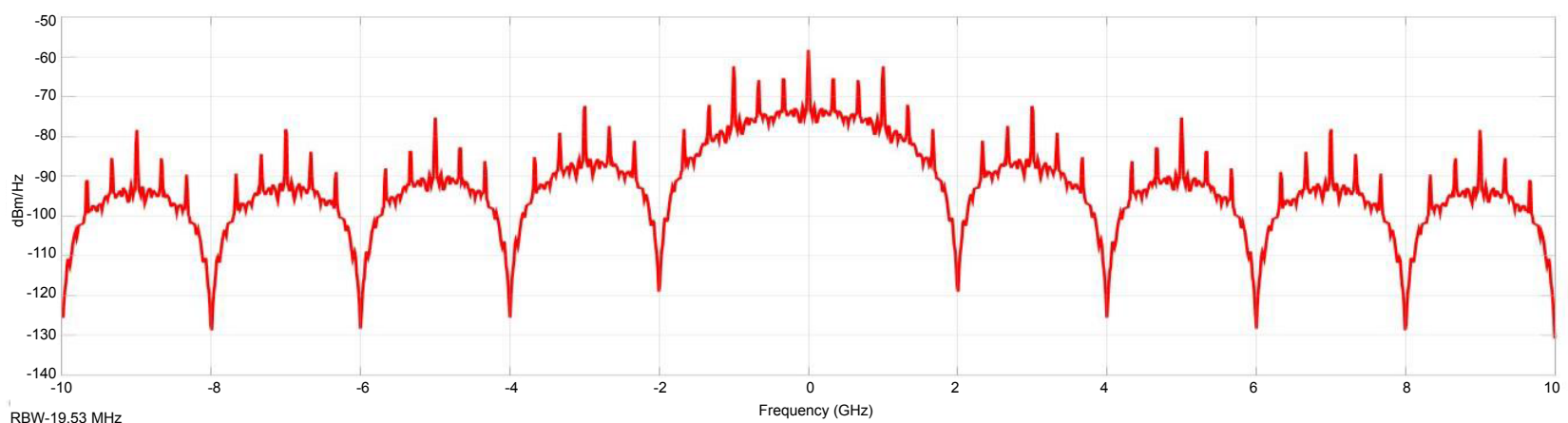

Figure 5: $P S D$ for $C C$ with $R=1 / 3$ and $K=7$.

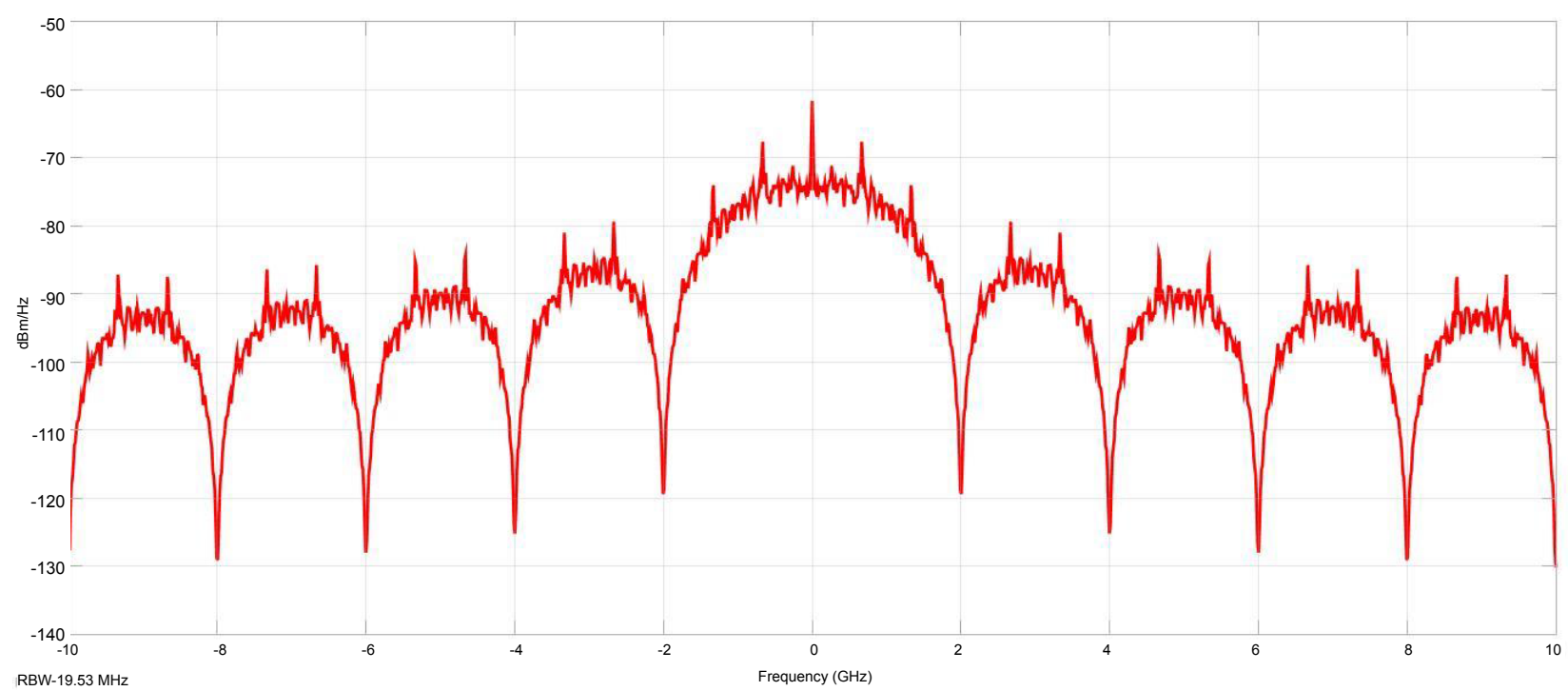

Figure 6a: $P S D$ for $T C$ with $R=1 / 3, L=100$ and $K=3$. 


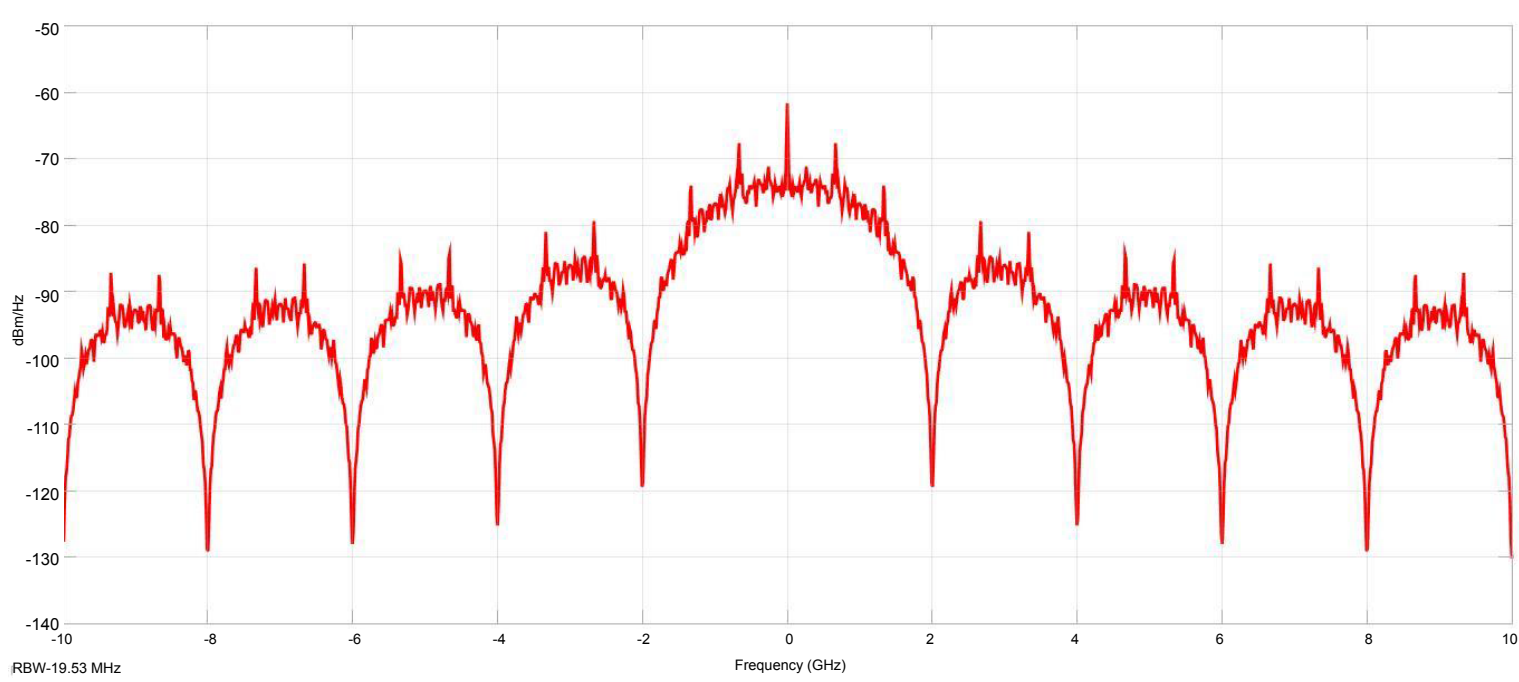

Figure 6b: $P S D$ for $T C$ with $R=1 / 3, L=10000$ and $K=3$.

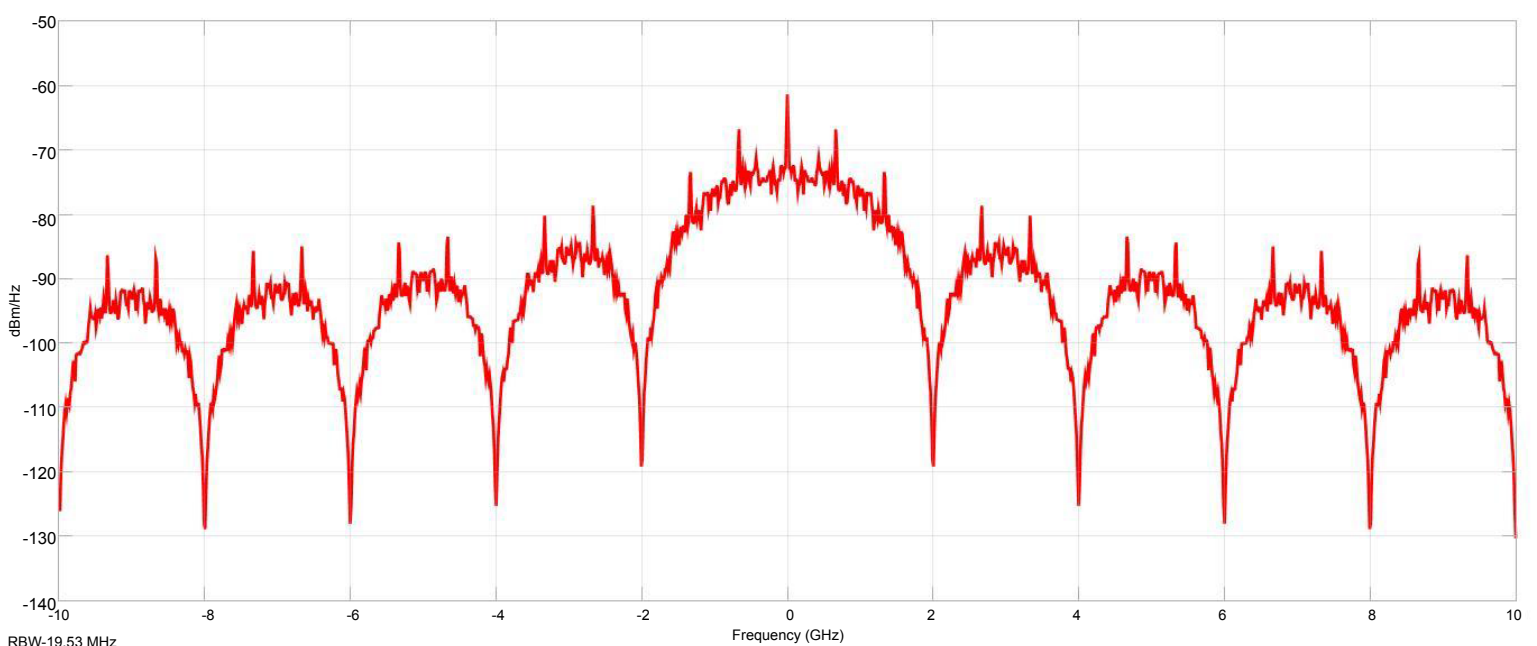

Figure 6c: PSD for $T C$ with $R=1 / 3, L=100$ and $K=5$.

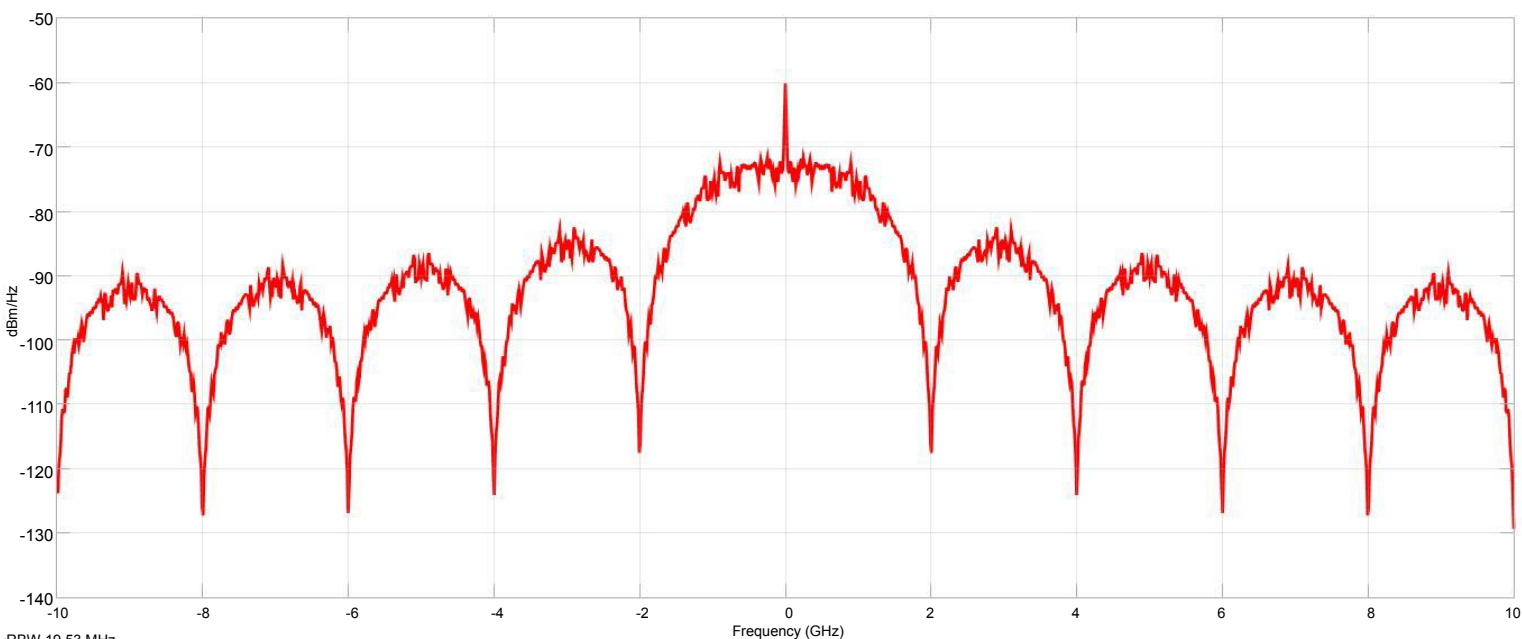

Figure 6d: $P S D$ for $T C$ with $R=1 / 3, L=10000$ and $K=5$. 


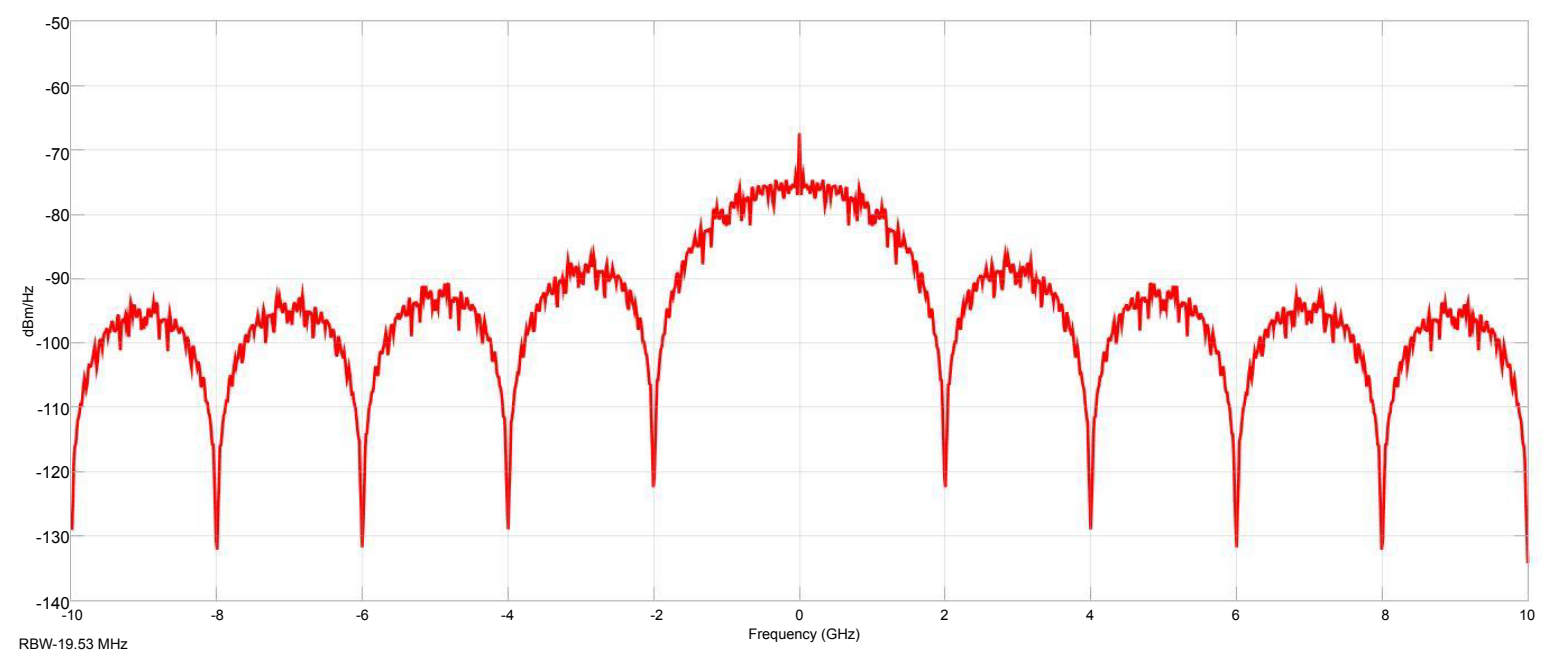

Figure 7a: PSD for MTC with $R=1 / 4, L=100$ and $K=3$.

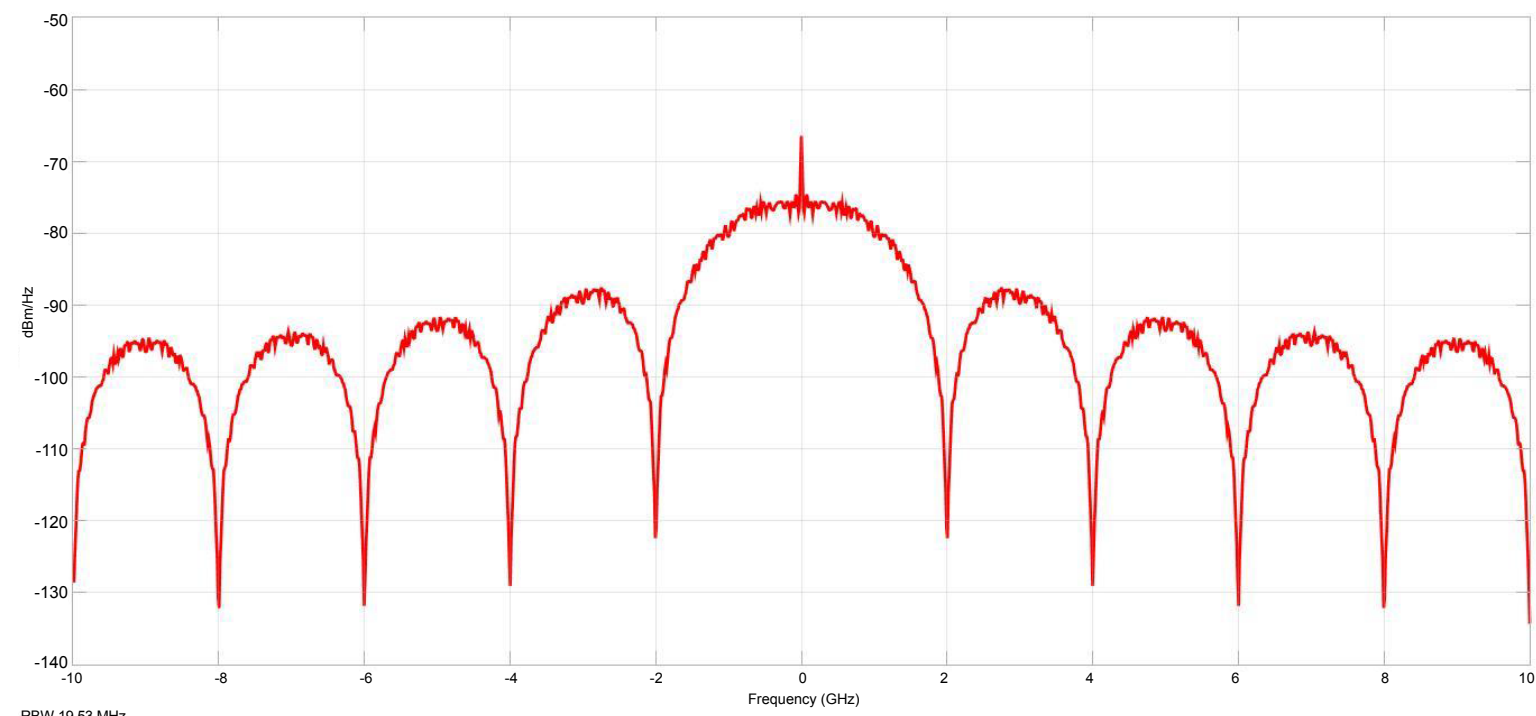

Figure 7b: PSD for MTC with $R=1 / 4, L=10000$ and $K=3$.

achieve the best performance in terms of suppression the spectral lines and makes it smoother.

\section{References}

1. FCC Notice of Proposed Rule Making (2002) Revision of part 15 of the Commission's Rules Regarding Ultra-Wideband Transmission systems. ETDocket, pp: 98-153.

2. Durisi G, Benedetto S (2004) Performance of Coherent and Non-coherent Receivers for UWB Communications. IEEE International Conference on Communications 6: 3429-3433.

3. Idriss A, Moorfeld R, Zeisberg S, Finger A (2005) Performance of Coherent and Non-coherent Receivers of UWB Communication, Second IFIP International Conference on Wireless and Optical Communications Networks, 2005. WOCN 2005.

4. Emami S (2013) UWB Communication Systems: Conventional and $60 \mathrm{GHz}$.

5. Molisch AF (2009) Ultra-Wideband Communications: An Overview. Radio Science Bulletin 2009: 3142

6. Oppermann IJ, Hamalainen M, linatti J (2005) UWB: theory and applications John Wiley \& Sons.
7. Senad C (2007) Application of UWB Technology for Positioning, a Feasibility Study.

8. Reddy SVN, Koppala N (2013) Design of Convolutional Codes for varying Constraint Lengths. International Journal of Engineering Trends and Technology 4: 61-66.

9. Proakis JG, Salehi M, Zhou N, Li X (1994) Communication systems engineering Englewood Cliffs: Prentice-hall, p: 889.

10. Liu Er (2004) Convolutional coding and Viterbi algorithm. IEEE, pp: 11-16.

11. Rana N, Qasem N (2015) Enhancing the Power Spectral Density of PPMIR for Ultra-Wide Band signals by using a convolutional encoder. In Internet Technologies and Applications (ITA), IEEE, pp: 14-17.

12. Divsalar D, Pollara F (1995) Turbo Codes for Deep-Space Communications. TDA Progress Report pp: 42-120.

13. Reyes V, Edwards RM, Al-haj Moh'd B (2006) On for the Comparison of Measurement and Simulation of the Power Spectral Density of PPM THIR UWB Signals. Loughborough Antennas and Propagation Conference Proceedings (LAPC) 2006, pp: 118-122. 
Citation: Jabbar M (2018) Improving PSD of PPM-IR for UWB Signal Using Turbo Encoder. Ind Eng Manage S3: 004. doi: 10.4172/2169-0316.S3-004

Page 6 of 6

14. Win MZ (2002) Spectral density of random UWB signals. IEEE Communications Letters 6: 526-528.
15. Reyes SR, Edwards RM (2004) Spectral Line Suppression in TH-IR Ultra Wideband Systems. 5th IEE International Conference on Mobile Communications Technologies (3G 2004). 